\title{
Handbook of Oral Cancer Screening \& Education: Prof Chittaranjan Choudhry
}

\author{
Anjan Shah
}

Received: 24 April 2011/Accepted: 30 April 2011/Published online: 17 May 2011

(C) Association of Oral and Maxillofacial Surgeons of India 2011

It has been many years since a book on oral cancer screening has been written and published. The last book on this theme I can recollect is Tobacco related oral mucosal lesions \& conditions in India-by Fali Mehta.

Oral cancer, as it increasingly presents in India is a difficult disease to treat surgically, and preventive measures are the only way to make an impact on this disease's progress. This is the book's main focus.

The book mainly focuses on the use of KAP (Knowledge, Attitude and Practice) to screen and counsel patients on their tobacco use and it's malignant potential.

The Oral \& Maxillofacial surgeon reading this book may find some of the epidemiological material published overwhelming, but most of the guidelines and review of literature regarding anti-tobacco measures are relevant to the surgeon, who may be the first person to initiate preventive therapy.
The book starts by describing the history and role of tobacco in the Indian subcontinent and its effects. It then describes in detail the KAP protocol that the author has initiated in collaboration with A B Shetty Memorial Institute of Dental Sciences in Mangalore and its results. Finally the book describes a quick screening protocol and has illustrations of tobacco related oral lesions and various types of quid.

I hope the reader of this book will be inspired to engage other medical \& dental colleagues as well as social organisations to use this book. This book is a useful guide to spread education on tobacco and oral cancer prevention among these colleagues.

Lastly, although the information published is excellent, there are a number of grammatical and text errors, which I hope will be corrected in the next proof reading.

\footnotetext{
A. Shah $(\bowtie)$

Bhagwan Mahaveer Jain Hospital, Vasanthnagar,

Bangalore 560052, India

e-mail: nongha1@yahoo.co.uk
} 